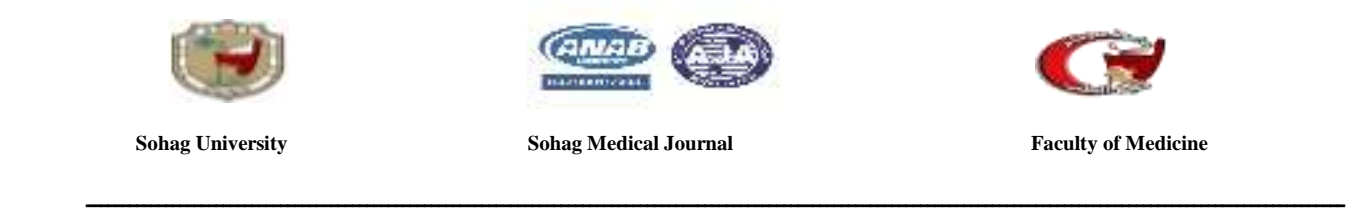

\title{
Outcomes of Non-Invasive laser Therapy for Treatment of Female Stress Urinary Incontinence
}

\author{
Hassan Ali Abdelhaleem, mohammed saber khalaf, Elnisr Rashid \\ Mohamed, Mohammed Dyaa S.saleem \\ Department of Urology, Sohag Faculty of Medicine, Sohag University
}

\begin{abstract}
Objective: Female stress urinary incontinence (SUI) is a common lower urinary tract symptom. $40 \%$ of them have mixed incontinence. we aimed to determine the effect of the type of urinary incontinence in laser outcomes. Study design: This was a prospective non-randomized study for female patients with SUI who were referred to the Urology Department, Sohag University Hospital from March 2017 to June 2018. The study was approved by the Sohag University ethics committee. Patients were treated by the clinical protocol for SUI with non-ablative $2940 \mathrm{~nm}$ Er: YAG laser. Patients were evaluated at the baseline and every 3 months for 1 year by Validated International Consultation on Incontinence Questionnaire - Urinary Incontinence Short Form (ICIQ-UI SF) Arabic version and the number of daily pads usage. Side effects of the treatment were also reported. Results: Our results included 32female patients. 20 patients (63\%) had SUI while 12 patients (37\%) had mixed incontinence. the median duration of symptoms 3 years. with 17 patients (53\%) had mild to moderate SUI. There was a significant reduction in daily pads usage with $P$ value $(<0.0001)$. and ICIQ-UISF score ( $p$ value $<0001)$. No serious side effects were reported. there was a statistically significant effect of the type of UI on laser therapy

outcomes. Conclusions Non-ablative Er: YAG laser therapy for SUI is a promising minimallyinvasive safe treatment alternative for SUI with no serious side effects. However, there was a statistically significant effect of the type of UI on laser therapy outcomes with better results related to pure SUI.
\end{abstract}

Keywords: SUI, UI, MUI, LASER, incontinence

\section{Introduction:}

Female stress urinary incontinence (SUI) is an important and common lower urinary tract symptom, middle-aged and elderly women are most commonly affected $^{(1)}$. It is known as "the symptom of involuntary urinary leakage related to effort or exercise, laughing, coughing or sneezing ${ }^{(2)}$. Urine loss can also occur during the sexual act and lead to significant distress ${ }^{(3)}$.
The prevalence of urinary incontinence is $16 \%$ for women younger than 30 years and $29 \%$ for older women (4). Risk factors of SUI and other pelvic floor dysfunctions are aging, pregnancy, route of delivery, smoking, obesity, and diabetes ${ }^{(5)}$. SUI may be due to the relaxation of the anatomical structure that supports the periurethral tissue or impairment of the function of the urethral sphincter. Women with SUI 
SOHAG MEDICAL JOURNAL

Vol. 24 No. 1 Jan 2020

have an altered connective tissue characterized by decrease collagen production, which may result in inadequate support of the urogenital tract (6).

Nonsurgical options for SUI include weight loss for obese patients, stop smoking, pelvic floor muscle exercise, Electrical stimulation, weighted vaginal cones and medication ${ }^{(7)}$. Surgical procedures such as Burch colposuspention are effective than nonsurgical options But they are associated with side effects and operative complications, for example bleeding, perforation of the bladder, injury of urethra, infection and chronic pelvic pain (8). The medical effects of lasers are well established. Thermal energy from the laser stimulates new collagen formation in addition to the immediate tightening of collagen fibrils by two-thirds of their length in comparison to the pretreatment state. A minimally-invasive, non-ablative Er: YAG laser vaginal tightening procedure can be used for this purpose.

Materials and methods: this is a prospective non-randomized study for female patients with SUI who were referred to the Urology Department; Sohag University Hospital during the period from March 2017 to June 2018. The study was performed after approval from Sohag University Hospital's ethics committee. The inclusion criteria of the study included Mild to moderate stress urinary incontinence by clinical evaluation then all patients were reevaluated according to ICIQ-UI SF score, Age of the patients more than 18 years, any number of parity and With or without mild prolapse (stage 1 and stage 2 by POP-Q system). The exclusion criteria were; neurogenic bladder,
Pregnancy, active genitourinary tract infection, Previous incontinence surgery, and severe pelvic organ prolapse. According to previous criteria, the study included 32 female patients.

The sample size was calculated using Power Analysis and Sample Size software program (PASS) version 15.0.5 for windows (2017) with the proportion of patients expected to be dry (ICIQ-UI SF score $=0$ ) after treatment as the primary outcome. A sample of 32 patients is needed to achieve $80 \%$ power (1- $\beta$ or the probability of rejecting the null hypothesis when it is false) with a significance level of $5 \% \quad(\alpha$ or the probability of rejecting the null hypothesis when it is true).

The protocol of management: All patients underwent a detailed medical history including, duration and severity of incontinence episodes, number of pads usage, menstrual and obstetric history, medical and drug history and any previous treatment modalities. The degree of SUI and its impact on the quality of life was assessed using the (ICIQ-UI SF), this questionnaire has been validated in Arabic. ${ }^{(10)}$ The ICIQUI SF score was classified into fourdegree: mild degree (1-5), moderate degree (6-12), severe degree(13-18) and very severe degree (19-21), as reported by Klovning et al. ${ }^{(11)}$

The full gynecological examination was done to determine the suitability of the patient for the vaginal laser therapy and we assessed the presence of urine leaks by cough test, POP, vaginal mucosal atrophy and any sign of infection or vaginal lesions. Patients were advised to perform a pregnancy test the day before the session to exclude pregnancy. Patients fulfilled the inclusion criteria were managed with at least one session of laser with time interval at least one 
month between sessions as the maximum effect of laser therapy need at least one month.

the patients were treated according to the clinical protocol using Er: YAG (2940 $\mathrm{nm}$ ) laser using the thermal only mode through a three-step protocol. The treatment was performed in the outpatient clinic with neither preoperative anesthesia nor analgesia or post-procedure medications. The procedure consisted of three phases. In the first step, the whole vaginal wall using the R11 full-field headpiece with a circular adaptor. In the second step, the anterior vaginal wall was irradiated using the PSO3 headpiece with an angular adaptor. Then The speculum was removed and the third step was done by the PS03 handpiece with a straight adaptor to irradiate both the mucosa of the vestibule and the introitus.

LASER TYPE Er: YAG (2940 nm)laser system (XS Dynamis, Fotona, Slovenia). Mode non-ablative thermal only SMOOTH mode" (energy $10.0 \mathrm{~J} / \mathrm{cm} 2$; four pulses per packet, pulse duration $250 \mathrm{~ms}$ with spot size $7 \mathrm{~mm}$ and rate 1.6 $\mathrm{Hz}$.

The improvement was defined as any reduction in The ICIQ-UI SF score and any reduction in the number of pads usage by the patients at 1,3,6 months and one year. However, the cure was defined as (ICIQ-UI SF) equal zero and failure was defined as no change in (ICIQ-UI $\mathrm{SF}$ ) or the number of pads usage. Patient's satisfaction with the treatment was also reported, using an ordinal scale: high satisfaction, moderate, low or no satisfaction.

Following the procedure, patients were observed for complications such as infection, pain, vaginal burn, urinary retention, loss of efficacy or complete failure. All patients were consulted to avoid sexual activity for at least one week, intravaginal tampons and no healing cream. Post-operative evaluation at 1, 3, 6 months and 1 year through (ICIQ-UI SF), number of pads and physical examination.

Statistical analysis: IBM's SPSS statistics were used for the statistical analysis of the collected data. Continuous variables were expressed as mean and standard deviation and median and inter-quartile range while categorical variables were expressed as number and percentage. Dependent samples Student t-test and Wilcoxon's signed-ranks tests were used for comparison of normally and abnormally distributed continuous data respectively. All tests were conducted with a $95 \%$ confidence interval. Bivariate correlations were assessed using Pearson's or Spearman's correlation coefficient depending on the nature of data. statistically, significance was considered when $\mathrm{P}$ (probability) value $<0.05$

\section{Results:}

The study included 32 female patients. With the median age of 38years (range 26-62 years) the median BMI per patient was 24 (range: 19-29.5). The median delivery number was 4 per patient (range: 2-8). Most of the patients were in premenopausal state $87 \% \quad(28$ patients). Twenty patients $(63 \%)$ had SUI while twelve patients $(37 \%)$ had mixed incontinence with a predominance of SUI. the median duration of symptom 3 years (range 1-5 year), Most of them were associated with pelvic organ prolapse of a low stage in about 29 patients $(91 \%)$ and as regards the severity of SUI 17 patients $(53 \%)$ had mild to moderate SUIas classified by ICIQ UI SF score. the median baseline 
SOHAG MEDICAL JOURNAL

Vol. 24 No. 1 Jan 2020

of pads usage was three pads per day

(rang 0-5) (Table 1).

\begin{tabular}{|c|c|c|c|c|c|}
\hline & \multicolumn{5}{|c|}{ All patients $(n=32)$} \\
\hline & Mean \pm SD & Median & IQR & Minimum & Maximum \\
\hline Age & $38.81 \pm 7.59$ & 38 & $34.25 ; 42.75$ & 26 & 62 \\
\hline BMI & $23.95 \pm 2.78$ & 24 & $22 ; 26$ & 19 & 29.5 \\
\hline Parity & $4.09 \pm 1.53$ & 4 & $3 ; 5$ & 2 & 8 \\
\hline $\begin{array}{l}\text { Normal vaginal } \\
\text { delivery }\end{array}$ & $3.66 \pm 1.45$ & 4 & $3 ; 5$ & 0 & 7 \\
\hline Abortion & $0.22 \pm 0.49$ & 0 & $0 ; 0$ & 0 & 2 \\
\hline $\begin{array}{l}\text { Duration } \\
\text { (years) }\end{array}$ & $2.28 \pm 1.28$ & 2 & $1 ; 3$ & 1 & 5 \\
\hline $\begin{array}{l}\text { Number of } \\
\text { daily pads }\end{array}$ & $2.84 \pm 1.53$ & 3 & $2 ; 4$ & 0 & 5 \\
\hline Postmenopause & \multicolumn{5}{|c|}{$13 \%(4)$} \\
\hline \multirow[t]{2}{*}{ Complaint } & SUI & \multicolumn{4}{|c|}{$63 \%(20)$} \\
\hline & Mixed UI & \multicolumn{4}{|c|}{$37 \%(12)$} \\
\hline \multirow[t]{3}{*}{ POP stage } & $\mathbf{0}$ & \multicolumn{4}{|c|}{$9 \%(3)$} \\
\hline & 1 & \multicolumn{4}{|c|}{$47 \%(15)$} \\
\hline & 2 & \multicolumn{4}{|c|}{$44 \%(14)$} \\
\hline
\end{tabular}

Table(1)

Demographic data, Obstetric and gynecological history of the study participants:
All patients were treated by laser therapy with a median number of the session was 2 sessions there was a significant reduction in the number of daily pads usage and the ICQI-UI SF score after the first session and as well as the number of session increase. However, this was obvious until the third session with no more reduction was seen after the third session. There was a significant reduction in the median of daily pads usage from a median of 3 pads per day (rang 0-5) at baseline to a median of one pad per day at both 6 months and one year (rang 0-3 pads per day) which was statistical significant at both 6 months and one year with $\mathrm{P}$ value $(<0.0001)$. By comparison of the daily pads usage and the degree of SUI there was significant post-treatment decrease in the number of pads usage as the degree of SUI increase with marked reduction related to severe cases with p-value $(<0.002)$.

ICIQ-UI SF median score before treatment was 12 (range: 5-16). Three patients (9\%) had mild degree SUI, 14 patients (44\%) moderate degree and 15 patients $(47 \%)$ severe degree. The postoperative media score of ICIQ-UI SF was 7 (range: 5- 16) at 3rd month, 5 (rang 0-16) at 6 months and 5.5(rang 014) at one year. The difference between the two scores (pre and post-treatment) per patient was significantly different (pvalue $<0.001$ ). and we reported a cure (no SUI symptoms and ICIQ-UI SF=0) in five patients (16\%) and improvement in overall score in 26 patients (81\%). By comparison of pre- and post-treatment ICIQ- UI SF score, the significant reduction in the ICIQ- UI SF score was due to improvement in the degree SUI till 6months at 7th month the effect of 
SOHAG MEDICAL JOURNAL

Vol. 24 No. 1 Jan 2020

laser therapy start to decrease as duration increase (figure 1).

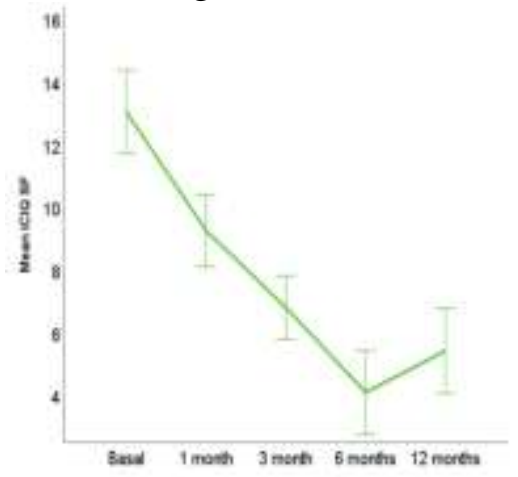

(Figure 1)Follow-up of the ICIQ UI-SF score throughout the post-treatment follow up period:

Seven patients $(22 \%)$ of patients reported high satisfaction with the results of the treatment, ten patients (31\%) reported moderate satisfaction with the results of the treatment and nine patients $(28 \%)$ reported mild satisfaction. Only $19 \%(n=6)$ were not satisfied with the treatment.

There was no significant effect of the type of UI on the number of sessions needed by patients to achieve clinical improvement. However, there was a statistically significant effect of the type of UI on laser therapy outcomes (Table 15).On the other hand, there was a significant correlation between the type of UI and predictor of the success of laser therapy such as daily pads usage, duration of symptoms, parity, and the number of vaginal delivery (Table 16).

\section{Discussion}

Surgical options are effective than nonsurgical options for treatment of SUI But they are associated with side effects and operative complications, for example bleeding, perforation of the bladder, injury of urethra, infection and chronic pelvic, tape erosion, urethral injury, infection, and chronic pelvic pain (8). As laser therapy offers patients' quality of life improvement, because it is minimally invasive and associated with no serious complications compared to mid-urethral slings So, it is considered a promising alternative option. However, there is a deficiency in randomized clinical trials (RCT) and studies comparing any laser procedure to the other well-established UI treatment options, such as pelvic floor exercises or mid-urethral slings.

In our study we used a validated questionnaire ICIQ-UI SF (Arabic version) and the number of daily pads usage per patient to report our results of Er: YAG laser therapy. The Arabic version is simple, easily applicable by Arabic patients and it was the first time to use an Arabic version for this purpose. This validated questionnaire was used to compare pre and post-treatment patient's status which revealed a significant improvement in SUI not only in terms of frequency and quantity but also the degree of patients' quality of life affection. Moreover, we used the number of daily pads usage for evaluation of improvement of SUI symptom which also the first time to be used for this purpose as an easy and applicable subjective method. Other studies report their results using other validated questionnaires, pad weight test, Q-tip test, urodynamics parameter or histological character of tissue which are objective methods but invasive and were time-consuming and not accurate especially for pads weights test. Overall, the clinical benefit of calculating the amount of urinary leak was unestablished to be used as a predictive value for treatment outcomes. ${ }^{(12)}$

The results of our study showed that laser effectively reduces the symptoms of SUI. And the improvement was evident after the first session, as reported 
by the patients before doing the second session and durable for at least 6 months. However, the efficacy decrease as duration increase post-treatment mainly after one year and may need another booster session as suggested by Gasper et al (2016) who reported that Treatment effects of laser sessions for at least three sessions with monthly intervals last about one year after these time the effect begins to decrease, but can be regained and maintained by " one maintenance session" every 6 months ${ }^{(13) .}$

The laser procedure was well tolerated by the patients and they were moderately satisfied with the clinical effect of laser therapy with no significant adverse effect or complications were observed except for decease efficacy as posttreatment duration increase and mild pain/discomfort during laser sessions which comparable to other studies (Fistonić et al and Pardo et al 2016) (14, 15). No patient was reported to stop laser procedure due to intolerance.

we reported a $16 \%$ cure rate (patients were dry (ICIQ-UI SF $=0$ ) at one year which is comparable to a $21 \%$ cure rate in a patient treated with laser in a randomized controlled trial with follow up only for 3 month patients. ${ }^{(16)}$ Tien et al 2017 also reported a $21.9 \%$ cure rate and a $12.5 \%$ improvement rate based on various questionnaires and scales at 6 months follow-up (17). However, Gaspar et al.2107 reported the cure of $64 \%$ and $46 \%$, respectively at the 3rdand 6th months of follow up of patients post laser therapy procedure ${ }^{(18) .}$

Improvement of SUI symptoms in our study was observed in $81 \%(n=26)$ of all patient which is comparable to the study did by Ogrinc et al 2015. who reported an improvement in $77 \%$ of patients after follow up of his patients for one year after the laser procedure. ${ }^{(19) .}$
Improvement in ICIQ-UISF score also reported by Gambacciani et al (2015) who perform his laser study in patients suffering from the genitourinary syndrome of menopause ${ }^{(20)}$. Also, Pardo et al (2016) showed significant improvement in ICIQ-UI SF scores and demonstrate that improvement occurred in $78.6 \%$ of his patients and while $38 \%$ showed complete cure and $16 \%$ no change (14). Fistonić et al,2016 also reported a significant reduction in ICIQUI SF score during the period of follow up with $(72.3 \%)$ of his patients who were reported to achieved improvement (15)

In our study, there was no significant effect of the type of UI (63\%SUI vs. $37 \%$ MUI) on the number of sessions needed by patients to achieve clinical improvement. However, there was a statistically significant effect of the type of UI on laser therapy outcomes. Which was comparable to A prospective nonrandomized study followed 175 patients suffering from SUI (66\%) and MUI (34\%) reported by Ogrinc et al (2015) and Follow-up was arranged at 2, 6, and 12 months. Who reported results at 1year-followup, (62\%) from both SUI and MUI groups reported no incontinence. In patients with SUI, the treatment statistically significantly improved in $77 \%$ of the cases, while the patients diagnosed with MUI before the treatment only improved in $34 \%$ of the cases ${ }^{(19) \text {. }}$

our study has some limitations, for example the short follow up period, the absence of control group or randomization, the small sample size and the absence of objective outcome measures .however, the data which were reported by using ICIQ UI SF score demonstrates that non-ablative Er: YAG laser therapy offers a novel and 
SOHAG MEDICAL JOURNAL

Vol. 24 No. 1 Jan 2020

minimally invasive option for treating of stress urinary incontinence with no long term serious side effect.

\section{Conclusions}

Non-ablative Er: YAG laser therapy for treating SUI looks like to be a promising minimally-invasive and safe alternative treatment option for SUIleading to a significant reduction of SUI symptoms, that lasting at least for 6 months and lead to improvements in the quality of life of patients with SUI with no serious side effects.

Conflicts of interest: there were no conflicts of interest of any kind

\section{References}

1- Luber KM. The definition, prevalence, and risk factors for stress urinary Incontinence. Rev Urol 2004; 6(Suppl. 3):S3-9.

2- Abrams P, Cardozo L, Fall M, et al. The standardization of terminology in lower urinary tract function: report from the standardization sub-committee of the International Continence Society. Urology. 2003; 61(1):37-49.

3- Temml C, Haidinger G, Schmidbauer J, et al. Urinary incontinence in both sexes: prevalence rates and impact on the quality of life and sexual life. Neurourol Urodyn 2000; 19:259-71.

4- S. Hunskaar, K. Burgio, A. Diokno, et al. Epidemiology and natural history of urinary incontinence in women, Urology 62(Suppl. 1) (2003) 16-23.

5- D.A. Patel, X. Xu, A.D. Thomason, et al. Childbirth and pelvic floor dysfunction: an epidemiologic approach to the assessment of prevention opportunities at delivery, Am. J. Obstet. Gynecol. 195 (1) (2006) 23-28.

6- Wong MY, Harmanli OH, Agar M, et al. Collagen content of nonsupport tissue in pelvic organ prolapse and stress urinary incontinence. American journal of obstetrics and gynecology 2003; 189(6):1597-1599; discussion 1599-1600.

7- Gustaf C. Creating a gold standard surgical procedure: the development and implementation of TVT. Int Urogynecol J 2015; 26:467-9.

8- G. Novara, W. Artibani, M.D. Barber, et al., Updated systematic review and meta-analysis of the comparative data on cop suspensions, pubovaginal slings, and midurethral tapes in the surgical treatment of female stress urinary incontinence, Eur. Urol. 58 (2) (2010) 218-238.

9- Thomsen S. Pathologic analysis of photothermal and photomechanical effects of laser-tissue interactions. Photochemistry and photobiology 1991; 53(6):825-835.

10- Hashim H, Avery K, Mourad MS, Ghoniem G, et al.The Arabic ICIQ-UI SF: an alternative language version of the English ICIQ-UI SF. Neurourol Urodyn 2006;25(3):277-282.

11- Klovning A, Avery K, Sandvik H, et al. Comparison of two questionnaires for assessing the severity of urinary incontinence: the ICIQ-UI SF versus the incontinence severity index. Neurourol Urodyn 2009; 28:411-5.

12- Al Afraa T, Mahfouz W, Campeau L, et al. Normal lower urinary tract assessment in women: I. Uroflowmetry and post-void residual, pad tests, and bladder diaries. Int Urogynecol J 2012; 23:681-5.

13- Gaspar A, Brandi H, Gomez V, et al. Photo-thermal Er: YAG laser treatment of stress urinary incontinence -3 years follow-up. SIU 2016, Buenos Aires, 20- 2310. 36th Congress of Société Internationale d'Urologie 2016; 139063.

14- Pardo JI, Solà VR, Morales AA. Treatment of female stress urinary incontinence with Erbium-YAG laser in non-ablative mode. Eur $\mathbf{J}$ Obstet Gynecol Reprod Biol. 2016;204:1-4

15- Fistonić N, Fistonić I, and Guštek ŠF, et al. Minimally invasive, non-ablative 
Er: YAG laser treatment of stress urinary incontinence in women-a pilot study. Lasers Med Sci. 2016; 31(4):635-43.

16- Mija Blaganjea, Ivan Verdenika, Franja Pajkb et al. Non-ablative E: YAG laser therapy effect on stress urinary incontinence related to the quality of life and sexual function: A randomized controlled trial. European Journal of Obstetrics \& Gynecology and Reproductive Biology 224 (2018) 153158.

17- Tien YW, Hsiao SM, Lee CN, et al. Effects of laser procedure for female urodynamic stress incontinence on pad weight, urodynamics, and sexual function. Int Urogynecol J. 2017; 28: 469-76.

18- Gaspar A, Brandi H. Non-ablative erbium YAG laser for the treatment of type III stress urinary incontinence (intrinsic sphincter deficiency). Lasers Med Sci. 2017; 32(3):685-91.

19- Ogrinc UB, Senčar S, Lenasi H. Novel minimally invasive laser treatment of urinary incontinence in women. Lasers Surg Med. 2015; 47(9):689-97.

20- M. Gambacciani, M. Levancini, M. Cervigni, Vaginal erbium laser: the second-generation thermotherapy for the genitourinary syndrome of menopause, Climacteric 18 (5) (2015) 757-763 